\title{
AS PERCEPÇÕES DE TRABALHADORES SOBRE A PRÁTICA FISIOTERAPÊUTICA NA INCAPACIDADE PARA O TRABALHO
}

\author{
WORKERS' PERCEPTIONS ABOUT PHYSICAL THERAPY \\ PRACTICE IN WORK INCAPACITY
}

Ana Lúcia Gadelha De Moura (ORCID: 0000-0001-6982-8517)

Kátia Suely Queiroz Silva Ribeiro (ORCID: 0000-0002-5850-7176) ${ }^{1,2}$

Maria Cláudia Gatto Cardia (ORCID: 0000-0001-5866-860X) ${ }^{1}$

Bianca Araújo Barbalho (ORCID: 0000-0002-1854-3295) ${ }^{1}$

Merlayne Pâmela De Oliveira E Silva (ORCID: 0000-0003-0409-3913)

Robson Da Fonseca Neves (ORCID: 0000-0002-3889-560X) 1,2

Autor Correspondente

Ana Lúcia Gadelha De Moura

E-mail: analuciagadelhamoura@gmail.com

\section{RESUMO}

Objetivo: compreender a prática fisioterapêutica na incapacidade para o trabalho na percepção de trabalhadores. Método: trata-se de uma pesquisa qualitativa que utilizou a entrevista semiestruturada em profundidade com 14 trabalhadores metalúrgicos com incapacidade prolongada para o trabalho em consequência de doenças e/ou acidentes do trabalho, os quais foram selecionados no Centro de Referência em Saúde do Trabalhador (Cerest). Para análise dos dados, foi aplicada a Análise de Enunciação. Resultados: duas categorias emergiram do trabalho de campo: a primeira, práticas fisioterapêuticas na incapacidade para o trabalho, na qual abordaram-se os aspectos alívio das dores e a burocracia da reabilitação; a segunda, reflexões sobre a fisioterapia na incapacidade para o trabalho, em que se abordou a reinserção do trabalhador para o trabalho e para a vida cotidiana. Discutiu-se a necessidade de as ações de fisioterapia ultrapassarem o espaço da atuação assistencial. Conclusões: há necessidade de refletir a respeito das abordagens usadas na reabilitação fisioterapêutica da incapacidade para o trabalho. Para isso, sugere-se discutir mais essa temática na formação acadêmica e nos espaços de educação continuada, sobretudo no que se refere a adotar abordagens biopsicossociais nos processos de avaliação e intervenção fisioterapêutica nas práticas voltadas à incapacidade.

Palavras-chave: Reabilitação; Fisioterapia; Saúde do trabalhador; Pesquisa qualitativa.

\begin{abstract}
Objective: to understand physical therapy practice in the incapacity for work in the perception of workers. Methods: this is a qualitative research that uses an in-depth semi-structured interview with 14 metallurgical workers with prolonged incapacity for work as a result of occupational diseases and/or accident. Participants were selected at the Occupational Health Reference Center (Cerest). For data analysis, the Enunciation Analysis was applied. Results: two categories emerged from fieldwork: the first, physical therapy practices in the incapacity for work, in which the aspects of pain relief and rehabilitation bureaucracy were addressed; the second, reflections on physical therapy in the incapacity for work were addressed: the reinsertion of the worker to work and to daily life. Conclusion: there is a need to reflect on the approaches used in the physical rehabilitation of work incapacity. Therefore, it is suggested to discuss this issue more in academic education and in continuing education spaces, especially regarding the adoption of biopsychosocial approaches in the processes of evaluation and physical therapy intervention in the practices related to disability.
\end{abstract}

Departamento de Fisioterapia, Universidade Federal da Paraíba.

${ }^{2}$ Programas de Pós-Graduação em Fisioterapia, Universidade Federal da Paraíba.
Keywords: Rehabilitation; Physical therapy specialty; Occupational health; Qualitative research. 


\section{INTRODUÇÃO}

A incapacidade para o trabalho é compreendida como consequência de aspectos físicos, sociais, psicológicos, culturais e administrativos que impossibilitam o retorno ao trabalho e que necessitam da atuação, do olhar e do investimento de outros agentes para desenvolver planos e ações com a finalidade de abordar o trabalhador da maneira mais adequada ${ }^{1}$. Pode ser considerada um problema de saúde pública, pois o número de casos vem crescendo no mundo e no Brasil ${ }^{2}$, o que pode causar impacto não apenas na vida do trabalhador, mas também no convívio social e familiar, quiçá na economia dos países.

A problemática da reabilitação na incapacidade para o trabalho vem sendo discutida na literatura científica com relação a diversos aspectos, tais como: a Reabilitação Profissional ${ }^{3}$; a relação com os profissionais de saúde ${ }^{4}$; a motivação e a autoconfiança dos trabalhadores quanto à reabilitação ${ }^{5}$; a associação entre reabilitação e retorno ao trabalho ${ }^{6}$; a mudança na autopercepção do trabalhador ${ }^{7}$; o empoderamento de trabalhadoras ante o processo de reabilitação ${ }^{8}$; e a relevância do apoio ao trabalhador nos diversos ambientes e nos quais ele está inserido9. Porém, ainda é escassa a produção de conhecimento que trate da abordagem da fisioterapia na incapacidade para o trabalho, sobretudo estudos que explorem as questões relacionadas com as percepções de trabalhadores ${ }^{10}$.

A reabilitação da incapacidade visa, na prática, por meio de programas terapêuticos com abordagem biopsicossocial, diminuir e superar as limitações funcionais, emocionais e sociais, incluindo um olhar de vigilância em ambiente e processo de trabalho agindo sobre a incapacidade para o trabalho, com o intuito de que, reabilitado, o trabalhador retorne às atividades com saúde e segurança ${ }^{11}$. Para isso, faz-se necessário entender que o trabalhador com incapacidade para o trabalho se relaciona com vários sistemas: o assistencial, o de seguridade social, o de trabalho e emprego e o familiar ${ }^{1}$. Dentro desses sistemas, ele lida também com seus agentes, estre os quais está o fisioterapeuta.

Por isso, questiona-se: o fisioterapeuta tem se comprometido com abordagens biopsicossociais e com ações de cunho integral nos problemas relacionados com os trabalhadores com incapacidade? Como trabalhadores que sofrem com a incapacidade percebem as práticas fisioterapêuticas? À vista disso, este estudo buscou compreender a prática fisioterapêutica na incapacidade para o trabalho na percepção de trabalhadores afastados por doença, analisadas em uma perspectiva biopsicossocial.

\section{MÉTODOS}

Trata-se de uma pesquisa qualitativa, que busca alcançar com profundidade os sentidos no dito e no dizer dos trabalhadores $^{12}$, cujos informantes foram trabalhadores metalúrgicos com incapacidade prolongada para o trabalho em consequência de doenças e acidentes relacionados com o trabalho. Os trabalhadores foram contatados no Centro de Referência em Saúde do Trabalhador (Cerest) em uma capital do nordeste brasileiro. O número de trabalhadores que participaram das entrevistas foi de 8 mulheres e 6 homens, os quais tinham idades de 37 a 54 anos e com tempo descontinuado de tratamento na fisioterapia variando de 1 a 15 anos, pois foram incluídos trabalhadores com incapacidade temporária ou permanente para o trabalho com, pelo menos, um momento de afastamento do trabalho, com recebimento de auxílio previdenciário por doença ou acidente de trabalho. Dentre estes, foram excluídos aqueles que estavam com algum litígio relacionado com o tratamento fisioterapêutico e que não possuíam condições cognitivas ou de fala no momento da pesquisa. 
A entrevista em profundidade foi escolhida para coleta dos dados e consiste em questões baseadas em uma estrutura flexível, na qual o entrevistado discorre livremente ${ }^{13}$. O roteiro dessa entrevista continha uma questão central, "Conte-me a sua história de trabalho desde o dia em que começou a trabalhar até os dias atuais". Nesse relato, a experiência de fazer o tratamento fisioterapêutico foi capturada $\mathrm{e}$, à medida que o informante apresentava um tópico novo, este era explorado pelo entrevistador. Um questionário para coletar as informações pessoais e de trabalho dos participantes também foi utilizado. As entrevistas foram gravadas e transcritas na íntegra.

A análise do conteúdo foi adotada na modalidade da análise de enunciação. Esta se respalda no fato de que o discurso é dinâmico e se constrói no momento em que é enunciado; e o analista avalia nesse discurso ou entrevista as palavras explícitas ou a omissão delas, as incoerências, contradições e imperfeições ${ }^{13}$. De acordo com Minayo ${ }^{14}$, o método de análise de enunciação segue a seguinte metodologia: a) estabelecimento do Corpus ou Corpi da Análise: é o conjunto de entrevistas com que se vai trabalhar; b) preparação do material: nesse momento, o pesquisador transcreve cada entrevista, deixando uma margem para anotações, buscando identificar as categorias no conjunto de entrevistas, ou seja, ele analisa a frequência com que certos temas são abordados durante a fala do emissor; e c) etapas da análise: na qual cada entrevista é analisada com totalidade e singularidade. São identificados também os aspectos: o alinhamento ao coletivo e a dinâmica própria do discurso do indivíduo; o estilo; os elementos atípicos; e as figuras de retórica.

Rosa $^{13}$ descreve a etapa "c" acima da seguinte forma: i) a dinâmica do discurso, em que estão inclusas a lógica que examina as relações entre as proposições e a análise sequencial, que investiga as quebras no decorrer do discurso, com decorrente mudança de assunto ou estilo ao longo da entrevista; ii) a análise do estilo do discurso, composta pelas litanias, que são repetições próximas e as interpolações, como, por exemplo, as pausas; e iii) a análise de elementos atípicos e figuras de retórica.
Neste estudo, foram observados os aspectos éticos e suas implicações contidas na Resolução n ${ }^{\circ}$ 466/2012 do Conselho Nacional de Saúde. Foi garantido o anonimato dos participantes, por isso seus nomes foram substituídos por E1, E2, E3... em que cada numeral corresponde a um entrevistado diferente. $\mathrm{O}$ projeto que deu origem a este recorte foi aprovado sob o número de CAAE: 84187918.3.0000.5030.

\section{RESULTADOS E DISCUSSÃO}

Duas categorias emergiram nessa investigação: práticas fisioterapêuticas na incapacidade para o trabalho, e reflexões sobre a fisioterapia na incapacidade para o trabalho: a reinserção do trabalhador ao trabalho e à vida cotidiana.

\section{Práticas fisioterapêuticas na incapaci- dade para o trabalho}

Existia, por parte dos trabalhadores em processo de reabilitação, a necessidade de fazer e continuar fazendo a fisioterapia de forma quase indeterminada:

Faço fisioterapia, fisioterapia é constante na minha vida. Tipo assim eu não vou só quando tá trovoando, só pra você ter uma ideia. (E3)

Esse aspecto carrega alguns questionamentos, como: quais motivos levam essas pessoas a fazerem tanta fisioterapia? Qual a expectativa desses trabalhadores quanto ao plano terapêutico estabelecido? Dois aspectos - alívio das dores e Fisioterapia como parte da burocracia da reabilitação ante a Previdência Social - surgem como respostas iniciais a esses questionamentos. 
Alívio das dores

Como em quase todo processo de reabilitação, espera-se da fisioterapia um resultado positivo e significativo, em outras palavras, a cura. Porém, se a cura não acontece, as expectativas diminuem. Por consequência disso, surge então o "alívio", o qual é sempre mencionado no sentido de amenizar as dores e de promover conforto momentâneo com o tratamento.

A fisioterapia foi uma coisa boa porque alivia, não cura né... É uma coisa boa, é uma coisa boa, alivia entendeu (mexe a cervical), é um parceiro que você tem para aliviar toda a dor e todo o sofrimento que a dor lhe causa ela alivia. Ela não lhe cura, mas ela é bom alívio. (E12)

Os trabalhadores procuram a fisioterapia de forma processual ou pontual nos seguintes momentos: na fase inicial, quando a dor ainda é local; no processo de cronificação do quadro; no período do afastamento e após alguma intervenção cirúrgica. E13 diz: “[...] é um processo que antes de eu fazer cirurgia eu fazia fisioterapia e mesmo assim... Hoje eu fiz cirurgia tou fazendo fisioterapia [...]”. Esse processo suscita uma rotina diária de idas e vindas à fisioterapia gerando uma dependência imposta pela necessidade do alívio das dores.

Nesse cenário, os trabalhadores passam pelo processo de ter que se adaptar a uma rotina de tratamentos prolongados, com evolução lenta e recidivas, gerando frustração, desalento, falta de perspectiva com o tratamento e desesperança em relação à melhora do quadro clínico. $\mathrm{O}$ recorte de fala abaixo aponta isto, por meio da repetição do termo "tanto tempo", dando ênfase ao tratamento prolongado e da metáfora "que eu me demito" atestando o fato de ser uma prática exaustiva e, por isso, ela está sempre mudando de clínica, na tentativa de mascarar uma rotina de anos.
Rapaz, eu faço fisioterapia há tanto tempo, tanto tempo que eu me demito de um lugar pro outro. (E7)

Esse tempo extenso de tratamento pode diminuir a credibilidade quanto ao tratamento fisioterapêutico e nos leva a questionar sobre o olhar da fisioterapia para a saúde do trabalhador, no sentido de não se deslocar do seu lugar de cura e reabilitação física, de não problematizar o seu papel social diante da incapacidade para o trabalho e de não trazer para dentro do set terapêutico outras perspectivas para este trabalhador.

Sem dúvida, a dor é um objeto de intervenção da fisioterapia, são inúmeros os estudos que tratam de técnicas e procedimentos fisioterapêuticos para intervir nesse mal que assola as populações mundiais ${ }^{15}$. Na incapacidade para o trabalho, a dor também é descrita como um importante elemento a ser enfrentado no processo de reabilitação, portanto, também é objeto da fisioterapia. Contudo, olhar só para a dor como evento clínico isolado do mundo do trabalho parece ser insuficiente ${ }^{16}$.

O estudo de Lima et al. ${ }^{17}$ tratou da reabilitação de trabalhadores com Lesões por Esforços Repetitivos/ Distúrbios Osteomusculares Relacionados ao Trabalho (LER/Dort) e apontou que os que estão nesta condição têm períodos prolongados de afastamento do trabalho em consequência das implicações físicas e psíquicas que afetam tanto as atividades laborais quanto de vida diária. Assim, questões sociais, políticas e culturais, além da maneira como o trabalhador reabilitado é visto pela empresa - e como o trabalhador se sente dentro do ambiente de trabalho -, têm grande influência na motivação e no processo de reabilitação do trabalhador. Os autores afirmam ainda que o plano de reabilitação deve incluir o reconhecimento das barreiras e dos facilitadores para serem debatidos com os trabalhadores e os outros agentes envolvidos e, desta forma, incluí-los no processo tratamento-reabilitação ${ }^{2}$. 
da fisioterapia, quer seja dentro ou fora do ambiente de trabalho, continua voltada para a função e para a estrutura do corpo lesado. Uma forma de superar isso é passar a adotar a abordagem biopsicossocial que não nega o olhar sobre a incapacidade, mas focaliza também na funcionalidade. Nesse sentido, o uso da Classificação Internacional de Funcionalidade, Incapacidade e Saúde (CIF) como uma ferramenta para orientar cuidados e incorporar a perspectiva biopsicossocial para o trabalhador pode ser uma importante ferramenta. A CIF oferece uma linguagem que retrata a incapacidade e a funcionalidade relacionada com as condições de saúde, tirando o foco da consequência da doença (deficiência) e trazendo-o para a funcionalidade, aspecto positivo que coloca em relevo as potencialidades que podem ser trabalhadas para que o sujeito tenha uma participação social maior, ou seja, quando o ambiente é acessível e possui políticas sociais positivas, a incapacidade abre caminho para integração e participação social ${ }^{18}$.

A CIF tem como um dos principais objetivos apresentar uma abordagem funcional que não se limita apenas à patologia, mas avança para uma abordagem biopsicossocial que inclui barreiras e facilitadores ambientais em relação às limitações da participação social. A OMS tem empreendido esforços para tornar a CIF, para além do seu uso como classificação de consequências da doença, um instrumento útil para avaliação. Dessa iniciativa, surgiram o "checklist" e o "core set" como primeiras aproximações da CIF para quem atua na ponta. Assim, advoga-se aqui que o fisioterapeuta precisa conhecer mais e se apropriar da CIF não apenas como classificação, mas também aplicando seus princípios e conceitos para avaliação e gestão do cuidado ${ }^{19}$. Segundo Melo et al. ${ }^{20}, 63,6 \%$ dos fisioterapeutas que atuam no Cerest têm conhecimento prévio sobre a CIF. Contudo, $82,2 \%$ não utilizam a CIF como apoio para o tratamento-reabilitação da LER/Dort, e 79,8\% dos Cerest apontam a carência de participação prévia da equipe para capacitação e discussão sobre a CIF.

Essa é uma das barreiras para que se desenvolva uma abordagem singularizada que enxergue a reabilitação além da melhora física apenas. Uma vez que, as doenças ocupacionais apresentam distúrbios de outra ordem, como estresse, cansaço físico ou mental, fadiga, sobrecarga, pressão temporal e baixo nível de controle sobre o trabalho, a subjetividade da dor e a evolução clínica imprevisível interferem na definição de um plano de tratamento adequado e repercutem em longos períodos de tratamento. Consequentemente, requerem uma abordagem fisioterapêutica mais afinada com a abrangência das demandas que cada caso apresenta e dependem da relação que se estabelece entre o fisioterapeuta e o paciente, no sentido de dialogar com o paciente sobre o seu caso e estabelecendo com ele os objetivos de tratamento para que haja adesão proativa ao tratamento ${ }^{21}$.

Neves e Nunes ${ }^{22}$ sumarizam afirmando que a atuação da fisioterapia para o trabalhador lesionado segue duas direções: uma na qual os benefícios gerados pela fisioterapia, se feita de forma segmentada, auxilia apenas na diminuição ou alívio dos sintomas da doença; e outra na qual se nota a falta de sensibilidade por parte dos profissionais de fisioterapia diante de outras necessidades do processo de reabilitação desses trabalhadores, que poderiam demandar outras práticas.

\section{Fisioterapia como parte da burocracia da reabilitação ante a Previdência Social}

A reabilitação profissional era realizada, preferencialmente, por uma equipe multiprofissional na qual a fisioterapia estava inserida. Porém, com o desmontes dos Centros de Reabilitação Profissional (CRP), no final da década de 1980 e início década de 1990, a reabilitação profissional foi fragmentada e delegada ao Sistema Único de Saúde (SUS) ${ }^{11}$. Nesse modelo, não há diálogo interprofissional no entorno do trabalhador com incapacidade, o que se exige desse trabalhador é que apresente à Previdência Social os cartões de presença que atestem o comparecimento às sessões de fisioterapia e os relatórios que são entregues à perícia atestando que o trabalhador cumpriu sua parte perante esse processo. 
Esse fato leva alguns trabalhadores a compreenderem a fisioterapia como parte de um itinerário burocrático. Assim, os trabalhadores com incapacidade assumem uma posição, muitas vezes, acrítica e utilitarista da fisioterapia e usam-na como um meio para conseguir mais um documento, sem a reflexão sobre o efeito da fisioterapia na melhoria das condições de saúde.

Eu vou, eu vou toda munida de exames para perícia... O relatório da fisioterapia né falando que eu faço as fisioterapias para me tratar. Estou levando as receitas que eu preciso tomar dos remédios para complementar a fisioterapia. (E1)

$\mathrm{Na}$ análise do discurso de forma geral, e na análise de enunciação adotada aqui, as omissões e as ausências também são percebidas e analisadas. Assim, a falta de registros narrativos que apontem para inquietações dos fisioterapeutas sobre os cartões de presença e sobre os relatórios faz-nos inquerir: o fisioterapeuta tem refletido sobre seu papel efetivo no processo de reabilitação da incapacidade para o trabalho? O que, de fato, ele deseja informar sobre o processo de reabilitação? Por fim, o quanto o fisioterapeuta está apenas servindo para manter um sistema de reabilitação que apenas burocratiza o processo, em detrimento de uma reabilitação que, efetivamente, seja voltada para melhoria de vida e da saúde e segurança no trabalho? Defende-se, aqui, que a atuação do fisioterapeuta na reabilitação da incapacidade precisa dar voz ao trabalhador e dialogar com os demais sistemas envolvidos nessa questão ${ }^{1}$.

A fisioterapia pode entrar como parte fundamental na reabilitação do trabalhador com incapacidade, por meio de várias estratégias e instrumentos para auxiliar na melhora do quadro clínico do trabalhador e, além disso, discutindo com o trabalhador os aspectos relativos à independência funcional e à possibilidade de capacitá-lo para uma nova função. Para isso, é necessário que o fisioterapeuta conheça bem os impactos gerados pelas disfunções musculoesqueléticas e psicossociais na saúde do trabalhador ${ }^{1}$; buscando, a partir de então, planejar ações para abranger, notificar, tratar e readaptar o trabalhador, levando em conta fatores organizacionais, socioculturais e territoriais relacionados com as disfunções que o levaram a incapacidade.

Decerto que, mantidos os padrões atuais de concessão do benefício auxílio-doença acidentário, a fisioterapia continua presa à emissão de cartões de presença e relatórios para proteger o trabalhador, na relação deste com o sistema de seguridade social, que exige dele, de forma compulsória, esses documentos para a obtenção do benefício. Contudo, propõe-se aqui que, ao emitir tais documentos, ele leve em consideração que a reabilitação é parte de uma trajetória que se caracteriza pelo agravamento da doença e pela procura por assistência, pela legitimação da doença etc., e que, como agente que entende a reabilitação da incapacidade de forma ampliada, precisa também refletir sobre esses processos e incorporar nas suas práticas o diálogo com os trabalhadores sobre temas como vigilância sobre o ambiente, processo e organização do trabalho, e práticas de empoderamento individual e coletiva para lidar com os vários sistemas envolvidos na reabilitação da incapacidade para o trabalho, alçando-os a protagonistas desse processo ${ }^{22-24}$

\section{Reflexões sobre a fisioterapia na incapa- cidade para o trabalho: a reinserção do trabalhador ao trabalho e à vida cotidiana}

Os achados revelam que é importante repensar a atuação da fisioterapia na incapacidade para o trabalho. Nos relatos, observou-se que a fisioterapia traz benefícios além daqueles já destacados na reabilitação física. Esses serviços prestados pela fisioterapia operam, no nosso entendimento, na reintegração do trabalhador à vida cotidiana. Por conseguinte, notou-se que se o fisioterapeuta utiliza outras ferramentas - como escutar, enxergar potencialidades que o trabalhador pode explorar, integrar o tratamento aos objetivos de vida e não só de trabalho, incentivar as relações interpessoais entre as pessoas em tratamento, entre outras - isso, pode se traduzir em sensação de bem-estar, acolhimento, promoção da saúde e tratamento humanizado. Os recortes de fala 
abaixo evidenciam isso, em que, no primeiro recorte, o entrevistado usa a metáfora "fundo do poço" para caracterizar sua situação emocional antes da interação com o fisioterapeuta:

E só fui me afastando, me afastei de muita gente... um belo dia eu fui pra uma fisioterapia, encontrei um fisioterapeuta, ele me, me disse algo que eu disse assim, é eu ainda não, não morri, eu tô viva... Foi quando eu decidi seguir em frente. Eu me sinto útil. Mesmo muitas vezes não tendo condições de digitar [risos], mas eu tenho condição de pensar e isso foi o que me tirou lá do fundo do poço, foi essa palavra que eu ouvi do fisioterapeuta. (E7)

Então hoje eu faço na fisioterapia ombro, cervical e lá eu posso desempenhar várias atividades ergonômicas que exercitam a minha musculatura né que fazem com que eu tenha mais habilidade pra poder lidar no dia a dia e en encaro isso como uma forma boa. (E13)

O trabalhador com incapacidade para o trabalho passa um tempo fazendo fisioterapia, dentro do mesmo ambiente, e um bom profissional consegue aproveitar dessa circunstância para promover conforto, segurança e tranquilidade ao trabalhador. Contudo, a atuação da fisioterapia não precisa se limitar ao locus da clínica, do ambulatório, do hospital e de outros espaços, sua ação pode se expandir para o espaço da casa, do lazer, do estudo, da atividade religiosa, da atividade cultural e, quiçá, para o próprio trabalho. As orientações sobre hábitos e gestuais de vida diária, exercícios domiciliares, práticas de autorrelaxamento, adaptações ergonômicas, entre outras, podem ajudar no resgate do cotidiano e da independência funcional desses trabalhadores. Como observado no recorte de fala desse aspecto, o trabalhador traz a repetição da palavra "limitação" com intuito de dar ênfase à sua situação e como a fisioterapia traz benefícios perante essas limitações:

[...] sem a fisioterapia você acaba ficando mais limitado... porque ela consegue fazer com que você consiga melhorar esse campo de limitação entendeu. Então eu vejo a fisioterapia como uma coisa boa e ela entrou na minha vida nesse sentido de ajudar a melhorar essas limitações... ela me ajuda a melhorar o campo de limitações pra que eu possa conseguir fazer as coisas do meu dia a dia ou pelo menos parte delas né porque não consegue se fazer tudo. (E13)

A importância da fisioterapia na assistência das condições clínicas relacionadas com as doenças do trabalho já é bastante difundida e solidificada. No entanto, as práticas assistenciais vêm sendo feitas de forma desconectada do mundo do trabalho, por causa do modelo fragmentado de reabilitação atualmente adotado ${ }^{11}$. Por isso, defende-se que o fisioterapeuta deve ser um ator importante no empoderamento do trabalhador na busca de mudanças nos processos produtivos para assegurar ambientes de trabalho seguros e saudáveis. Desse modo, é preciso que o trabalhador se aproxime mais do conceito e das práticas inerentes ao campo da saúde do trabalhador, o qual considera o fenômeno saúde-doença no trabalho organizado nos eixos de: promoção da saúde e Vigilância em saúde do trabalhador (Visat), que compreende a vigilância epidemiológica dos agravos de doenças relacionadas com o trabalho e vigilância de processos e ambientes de trabalho, além da produção e divulgação de ações de educação em saúde ${ }^{25}$.

Assim, tomando como ponto de partida os elementos constituintes do campo da saúde do trabalhador descritos acima, urge pensar em novas práticas para ressignificar o lugar do fisioterapeuta e da fisioterapia na incapacidade para o trabalho. Primeiro, insta entender que a incapacidade para o trabalho se dá no curso da vida, ou seja, não é possível dissociar as práticas de reabilitação fisioterapêutica na perspectiva clínico-assistencial de outras práticas e vivências sociais como nas relações já citadas com os sistemas de saúde, de seguridade social, do trabalho e emprego e o familiar1. Segundo, o fisioterapeuta precisa munir-se de outro aparato de práticas que possam conferir ao trabalhador: empoderamento, autonomia, cooperação e protagonismo no seu processo de reabilitação.

Nesse sentido, duas alternativas que já existem na atenção primária e 
poderiam ser adotadas nos demais níveis de atenção pelo fisioterapeuta são: o acolhimento, o qual é fundamentado na escuta qualificada a fim de identificar se sinais, sintomas e/ou queixas estão relacionados com o trabalho atual ou pregresso ou outro aspecto da vida cotidiana que também interfere na saúde desses sujeitos; e os grupos operativos, que têm o propósito de realizar educação em saúde, formular ações preventivas e promover a corresponsabilização do usuário com o seu tratamento. As atividades em grupo são vistas como uma estratégia positiva já que abrem espaço para a escuta e compartilhamento de ideias $^{26}$.

Além disso, o fisioterapeuta deve incentivar a autonomia do trabalhador nas práticas de autocuidado na perspectiva da educação em saúde. Uma prática consolidada no campo da fisioterapia que promove esse tipo de cuidado é a Escola de Posturas $^{27}$. Esta consiste em um programa terapêutico para um grupo de pessoas, que inclui exercícios e instruções a respeito da postura ideal, abrangendo também o que pode estar relacionado com a postura, como aspectos sociais, ambientais e culturais com o intuito de amenizar problemas e discutir com os usuários formas de: cuidar e prevenir de maneira ativa dores e disfunções provenientes ou não da doença relacionada com o trabalho, hábitos de vida saudáveis, uso do conhecimento de ergonomia e biomecânica para a solução de problemas da vida cotidiana e no ambiente de trabalho ${ }^{28}$.

O fisioterapeuta é um dos profissionais que precisam repensar suas práticas levando em consideração a vantagem que possuem de terem contato longitudinal com os trabalhadores em reabilitação, podendo assim abordar aspectos do processo saúde-doença-trabalho e suas repercussões na vida cotidiana ${ }^{29}$.

Desse modo, é premente refletir sobre a prática fisioterapêutica, visto que seu contato longitudinal com trabalhadores em reabilitação sugere ações que extrapolem as fronteiras da assistência clínica ${ }^{29}$. Também, é mister refletir sobre a importância de o fisioterapeuta que assiste trabalhadores se aproximar mais dos conceitos e práticas da saúde do trabalhador, uma vez que o campo da saúde do trabalhador tem como objeto o processo saúde-doença em relação ao trabalho e à vida cotidiana, tendo o trabalhador como centro desse processo. Por isso, tem o intuito de criar intervenções que levem o trabalhador a apropriar-se do seu papel como protagonista desse processo $^{30}$.

\section{CONCLUSÕES}

A fisioterapia pode auxiliar o trabalhador no processo de reabilitação da incapacidade para o trabalho trazendo-o para a posição de protagonista nesse processo, promovendo um ambiente no qual ele se sinta cuidado e ativo na reabilitação, e resgatando a autonomia ao romper as barreiras da clínica, proporcionando educação, promoção e prevenção da saúde.

Assim, a fisioterapia pode figurar com um agente importante no campo da saúde do trabalhador na abordagem da incapacidade para o trabalho vista de forma abrangente e pela inter-relação que os sistemas de saúde, seguridade social, trabalho e emprego e familiar podem exercer sobre ela. Para isso, esse profissional carece de maior compreensão sobre a singularidade e a integralidade que envolve o trabalhador que precisa ser reabilitado. Mais que isso, urge como fundamental refletir a respeito das abordagens usadas na reabilitação fisioterapêutica. para tal, essa discussão precisa ser travada na academia, nos fóruns, seminários e outros espaços de formação e educação continuada e permanente para que, assim, possamos minimizar e superar a visão fragmentada e alcançar um modelo de atuação que se aproxime da perspectiva biopsicossocial.

A percepção da fisioterapia por trabalhadores com incapacidade para o trabalho, neste estudo, trouxe novos elementos para que se possa repensar a reabilitação nesses casos; contudo, ainda há uma lacuna a ser preenchida nesse conhecimento. Para tanto, sugerem-se mais pesquisas que avancem sobre as questões enfrentadas por trabalhadores em reabilitação da incapacidade e sobre práticas fisioterapêuticas que 
primem pela atuação voltada para a perspectiva biopsicossocial, para a vigilância em saúde do trabalhador e para a promoção da saúde na incapacidade para o trabalho.

\section{REFERÊNCIAS}

1. Bahia. Orientações Técnicas para ações de prevenção e manejo da incapacidade para o trabalho no SUS. Secretaria da Saúde do Estado. Superintendência de Vigilância e Proteção da Saúde. Diretoria de Vigilância e Atenção à Saúde do Trabalhador. SESAB/SUVISA/DIVAST. Salvador: DIVAST; 2014. 49p.

2. Lima MAG, AGM, Bulcão CMA, Mota EMCL, Magalhães FB, de Carvalho RCP, et al. Programa de reabilitação de trabalhadores com LER/DORT do Cesat/Bahia: ativador de mudanças na Saúde do Trabalhador Cesat/. Rev Bras Saúde Ocup. 2010;35(121):112-21.

3. Souza ADS, Queiróz MFF. Percepção dos trabalhadores inseridos na reabilitação profissional do Instituto Nacional do Seguro Social: a organização do trabalho adoece?. Saúde Debate. 2018;42(116):100-12.

4. O'Hagan FT, Coutu MF, Thomas SG, Mertens DJ. Work reintegration and cardiovascular disease: Medical and rehabilitation influences. J Occup Rehabil. 2012;22(2):270-81.

5. Åhrberg Y, Landstad BJ, Bergroth A, Ekholm J. Desire, longing and vanity: Emotions behind successful return to work for women on long-term sick leave. Work. 2010;37:167-77.

6. Mårtensson L, Hensing G. Experiences of factors contributing to women's ability to make informed decisions about the process of rehabilitation and return to work: A focus group study. Work. 2012;43:237-48.

7. Kvam L, Eide AH, Vik K. Understanding experiences of participation among men and women with chronic musculoskeletal pain in vocational rehabilitation. Work. 2013;45(2):161-74.
8. Ahlstrom L, Dellve L, Hagberg M, Ahlberg K. Women with Neck Pain on Long-Term Sick Leave-Approaches Used in the Return to Work Process: A Qualitative Study. J Occup Rehabil. Springer US; 2017;27(1):92-105.

9. Matérne M, Lundqvist LO, Strandberg T. Opportunities and barriers for successful return to work after acquired brain injury: A patient perspective. Work. 2017;56(1):125-34.

10. Maillette P, Coutu M, Gaudreault N. HPLC Bottom-Up MS-Based Proteomics for Mapping of Specific Proteins in Several European Spring Barley Varieties. J Am Soc Brew Chem. Elsevier Masson SAS; 2015;1-7.

11. Takahashi M, Kato M, Aylce R, Leite O. Incapacidade, reabilitação profissional e Saúde do Trabalhador : velhas questões , novas abordagens. Rev Bras Saúde Ocup. 2010;35(121):7-9.

12. Minayo MC de S. Análise Qualitativa: Teoria, Passos Fidedignidade.pdf. Cien Saude Colet. 2012;621-6.

13. Rosa PRDS. Uma Introdução a Pesquisa Qualitativa em Ensino de Ciências [Internet]. Campo Grande: UFMS; 2013. 172 p. Available from: http://www.paulorosa.docente.ufms.br/Uma_Introducao Pesquisa_Qualitativa_Ensino_Ciencias.pdf

14. Minayo MCS. O desafio do conhecimento - Pesquisa qualitativa em saúde. 9. ed. São Paulo: Hucitec; 2006. 304-318 p.

15. Wijma AJ, van Wilgen CP, Meeus M, Nijs J. Clinical biopsychosocial physiotherapy assessment of patients with chronic pain: The first step in pain neuroscience education. Physiother Theory Pract. 2016;32(5):368-84

16. Santana S, Tamanini G. O tratamento fisioterapêutico da dor nos cuidados paliativos Physiotherapy treatment of pain in palliative care. Ling Acadêmica. 2017;7(6):41-53.;

17. Lima MAG de, Neves R da F, Tironi MOS, Nascimento AMDN, Magalhães F de B. Avaliação da funcionalidade dos trabalhadores com LER / DORT : a construção do Core Set da CIF para LER/DORT. Acta Fisiátrica. 2008;15(4):229-35. 
18. Organização Mundial da Saúde. Classificação Internacional de Funcionalidade, Incapacidade e Saúde. São Paulo: Editora da Universidade de São Paulo, 2004. 238 p.

19. Castaneda L. International Classification of Functioning, Disability and Health (ICF) - way to Health Promotion. Rev Bras Cineantropom Hum. 2018;229-33.

20. Melo BF, Souza ACAG, Ferrite S, Bernardes KO. Atuação do fisioterapeuta nos Centros de Referência em Saúde do Trabalhador: indicadores das notificações dos Dort. Fisioter e Pesqui. 2017;24(2):136-42.

21. Dosea GS, Oliveira CDCC, Lima SO. $<$ b $>$ Percepção da qualidade de vida em portadores de distúrbios osteomusculares relacionados ao trabalho. Ciência, Cuid e Saúde. 2016;15(3):482 .

22. Neves RF, Nunes MO. From legitimation to (re-)signification: the therapeutic itinerary of workers with RSIs/WMSDs. Cien Saude Colet. 2010;15(1):211-20.

23. Zavarizzi CP, Alencar MCB. Afastamento do trabalho e os percursos terapêuticos de trabalhadores acometidos por LER/ Dort. Saúde Debate. 2018;42(116):113-24.

24. Maceachen E, Kosny A, Ferrier S, Chambers L. The "toxic Dose" of system problems: Why some injured workers don't return to work as expected. J Occup Rehabil. 2010;20(3):349-66.

25. Brasil. Ministério da Saúde. Saúde do trabalhador e da trabalhadora. Cadernos de Atenção Básica, n. 41. Brasília: Ministério da Saúde; 2018. 136 p.

26. Lucchese R, Vera I, Benício PR, Silva ADF, Munari DB, Fortuna CM. Uso Do Grupo Operativo Na Atenção Em Saúde: Revisão Integrativa. Cogitare Enferm. 2014;19(4):823-32.
27. Cardia MC, Duarte M, Almeida R, Lima V. Manual da Escola de Posturas. 3. ed. João Pessoa: Ed. Universitária/UFPB; 2016.

28. Heymans MW, van Tulder MW, Esmail R, Bombardier C, Koes BW. Back schools for non-specific low-back pain. Cochrane Database Syst Rev. 2004;(4):CD000261.

29. Medeiros FC, Costa LOP, Oliveira NFC, Costa LCM. Satisfação de pacientes que recebem cuidados fisioterapêuticos para condições musculoesqueléticas: um estudo transversal. Fisioter e Pesqui. 2016;23(1):105-10.

30. Minayo-Gomez C, Vasconcellos LCF, Machado JMH. A brief history of worker's health in Brazil's unified health system: Progress and challenges. Ciênc Saúde Colet. 2018; 23(6):1963-70. $\frac{10}{\text { De Moura et al }}$

Recebido: 09/11/2019

Aprovado: 25/09/2021 